\title{
Antibacterial activity of coumarine derivatives synthesized from 4-amino- 7-chloro-2-0xo-2H-chromen -3-carbaldehyde and comparison with standard drug
}

\author{
Aziz Behrami 1 \\ Kozeta Vaso 2 \\ 1 Faculty of Food Technology University of Mitrovica Isa Boletini \\ 2 Faculty of Natural Science Universoty of Tirana
}

\begin{abstract}
In present paper, we report the organic syntheses of three compounds from 4-Amino-7-chloro -2-oxo-2H -chromen -3carbaldehyde and describe the results of antibacterial activity of purified compounds.7-Chloro-4-[(2-hydroxy-benzylidene)amino]-2-oxo-2H-chromen-3-carbaldehyde(1a).4-[(2-Hydroxy-benzylidene)-amino]-7-(4-hydroxy-phenylamino)-2-oxo-2Hchromen-3-carbaldehyde (2a) ,[4-[(2-Hydroxy-benzylidene)-amino]-7-(4-hydroxyl-phenylamino)-2-oxo-chromen-3ylmethylene]-amino)-acetic acid (3a),have been synthesized and characterized using melting points , IR spectra , 1H-NMR and 13C-NMR spectra. The antibacterial activity of synthesized compounds and streptomycin and cefalexine at concentractions of $2 \mathrm{mg} / \mathrm{ml}, 3 \mathrm{mg} / \mathrm{ml}$ and $5 \mathrm{mg} / \mathrm{ml}$, have been evaluated against three strains of bacterial culture; Staphylococcus aureus, E.coli and Bacillus cereus. The compounds show bacteriostatic and bactericidal activity.
\end{abstract}

Keywords: Coumarine derivatives , antibacterial activity ,IR,1H-NM,13C-NMR, Streptomycine.

\section{INTRODUCTION}

Starting from 4-Amino-7-chloro -2-oxo-2H -chromen -3-carbaldehyde (a); derivatives (1a,2a,3a) are synthesized.Coumarin derivatives are large group of heterocyclic with oxygen as heteroatom.Coumarin is a chemical compound (specifically, a benzo- $\alpha$ -

pyrone) found in many plants notably in high concentration in the tonka bean ( Dipteryx odorata), vanilla grass (Anthoxanthum odoratum) , woodruff (Galium odoratum) , mullein (Verbascum spp), and sweet grass (Hierochloe odorata).Coumarine and their derivatives have shown varius biological activities. Their fame has come mainly from their antithrombic, antiinflammatory, vasodilatory, and antiviral activities. Other several coumarin derivatives have antimicrobial properties 9 ( Z.M.Nofal ;M.El-Zahar; and S.Abd El Karim), with reflux and condensation we have synthesize some new coumarin derivatives and to investigate their antibacterial activity against Staphylococcus aureus, E.coli and Bacillus cereus.The antibacterial activity of synthesized compounds is compared with antibacterial activity of Cefalexine and Streptomycine.

\section{EXPERIMENTAL SECTION}

\section{Experimental Chemistry}

7-Chloro-4-[(2-hydroxy-benzylidene)-amino]-2-oxo-2H-chromen-3-carbaldehyde (1a), 4-[(2-Hydroxy-benzylidene)-amino]7-(4-hydroxy-phenylamino)-2-oxo-2H-chromen-3-carbaldehyde (2a) , ([4-[(2-Hydroxy-benzylidene)-amino]-7-(4-hydroxylphenylamino)-2-oxo-chromen-3-ylmethylene]-amino)-acetic acid (3a) are synthesized.

\section{Measurement}

The identification of derivatives 4-hydroxy-chromen-2-one (1a,2a,3a), is made by using melting point, IR, $1 \mathrm{H} \mathrm{NMR,} \mathrm{13C}$ NMR spectra and elemental analysis. Melting point was determinated on a Electrothermal apparatus (Fisher Scientific 2555 ) in a open capillary tube and are uncorrected.Infrared spectra were recorded in $\mathrm{cm}-1$ for $\mathrm{KBr}$ pellts on a $\mathrm{FT}-\mathrm{IR}$ Shimadzu 8400S spectrophotometer with resolution $4 \mathrm{~cm}-1$. $1 \mathrm{H}$ NMR spectra were recorded on a Bruker UNITY plus-500 'NMR 1' spectrometer using DMSO-d6 as the solvent and TMS as the internal references standard $(\sigma=0,00$ $\mathrm{ppm})$.Chemical shifts are expressed in $\delta \mathrm{ppm}$.Mass spectra were taken on a LKB 9000 mass spectrometer. Element analysze was performed on a Perikin-Elmer $240 \mathrm{BCHN}$ analyzer.The purity of the compounds (synthesized) was routinely checked by TLC using Merck Kieselgel-60 (F-254) and benzene,toluene,glacial acetic acid (80:10:10)as mobile phase The spots were exposed in iodine vapour for visualization.

\section{Preparation of 7-Chloro-4-[(2-hydroxy-benzylidene)-amino]-2-oxo-2H-chromen-3- carbaldehyde (1a)}

For this synthesis is used as substrat in a $100 \mathrm{ml}$ flask mixed $5 \mathrm{~g}$ 4-amino-7-chloro-2-oxo-2H-chromen-3-carbaldehyde , $10 \mathrm{ml}$ Dioxane, $0.5 \mathrm{ml} \mathrm{Et} 3 \mathrm{~N}$

The mixture was refluxed at $100 \mathrm{oC}$ for $5 \mathrm{~h}$. The obtained crystals yellow are filtred and rinsed with ethanol and dried at room temperature.Recrystallization form absolute ethanol gave a yellow product of $80 \%$ yield, melting point 327 oC.

\section{(Scheme.1)}




\section{Preparation of 4-[(2-Hydroxy-benzylidene)-amino]-7-(4-hydroxy-phenylamino)-2-oxo-} 2H-chromen-3-carbaldehyde (2a)

In a $100 \mathrm{ml}$ flask were mixed 4g 7-Chloro-4-[(2-hydroxy-benzylidene)-amino]-2-oxo-2H-chromen-3-carbaldehyde with 10ml Ethanol , $3 \mathrm{~g}$ amino fenole. The mixture was refluxed at $90 \mathrm{oC}$ for ca. $6 \mathrm{~h}$.

Theobtained red crystals are filtred and dried at room temperature . Recrystallization form $\mathrm{C} 2 \mathrm{H} 5 \mathrm{OH}$ gave red crystals product of $70 \%$ yield,meltingpoint, 399 oC.

\section{(Scheme 2) .}

Preparation of ([4-[(2-Hydroxy-benzylidene)-amino]-7-(4-hydroxyl-phenylamino)-2-oxo-chromen-3-ylmethylene]-amino)acetic acid (3a)

In a $100 \mathrm{ml}$ flask were mixed $3 \mathrm{~g}$ of 4-[(2-Hydroxy-benzylidene)-amino]-7-(4-hydroxy-phenylamino)-2-oxo-2H-chromen-3carbaldehyde , with $10 \mathrm{ml}$ Ethanol. The mixture was refluxed at $100 \mathrm{oC}$ in water bath for $\mathrm{ca} .8 \mathrm{~h}$. The flask was placed in an ice bath for $1 \mathrm{~h}$ until yellow crystalline precipitate was formed.After filtration the product was recrystallized from $\mathrm{C} 2 \mathrm{H} 5 \mathrm{OH}$ .The recrystallizacion gave a red product at $70 \%$ yield, melting.point; 459 oC.

\section{(Scheme 3 ).}

\section{Table-1 Analytical data}

Compd m.p M.F Elemental analysis. Calculatet :Found (calc) \% C H N O Cl 1a 327 oC C17H10CINO4 62.30 62.28 3.08 3.00 4.27 4.2019.5319.5010.8 10.7

2a 399 oC C23H16N2O5 69.0068 .54 .034 .0076 .9519 .9819 .98

3a 459 oC C25H21N3O6 63.35 63.30 4.614.58 9.159.12 20.8920.87

Antibacterial activity

The purified synthesized compounds (1a,2a,3a) was subjected to test in vitro its antibacterial activity against three bacterial cultures ; Staphylococcus aureus,E.Coli and B.cereus. Antibacterial activity of compounds was investigated applying the Kirby-Bayer method or disc method ( $d=5.5 \mathrm{~mm}$ max. capacity $10 \mu \mathrm{g}$ )

\section{Table 2 Antibacterial activity- Staphylococcus aureus}

Inhibition zone $(\mathrm{mm})$

Compound $2 \mathrm{mg} / \mathrm{ml} 3 \mathrm{mg} / \mathrm{ml} 5 \mathrm{mg} / \mathrm{ml}$

1a 111517

2a 121619

3a 131620

Cephalexine 888

Streptomycine 202020

\section{Table 3 Antibacterial activity - E.Coli Inhibition zone (mm)}

Compound $2 \mathrm{mg} / \mathrm{ml} 3 \mathrm{mg} / \mathrm{ml} 5 \mathrm{mg} / \mathrm{ml}$

1a 71216

2a 81418

3a 91817

Cephalexine 888

Streptomycine 202020

\section{Table 4 Antibacterial activity - Bacillus cereus Inhibition zone ( $\mathrm{mm})$}

Compound $2 \mathrm{mg} / \mathrm{ml} 3 \mathrm{mg} / \mathrm{ml} 5 \mathrm{mg} / \mathrm{ml}$

1a 71217

2a 81318

3a 111719 
Cephalexine 999

Streptomycine 202020

\section{RESULTS AND DISCUSSION}

By reacting equimolar amounts of 4-Amino-7-chloro -2-oxo-2 $\mathrm{H}$-chromen -3-carbaldehyde and correspomding reagents (according scheme 1) under reflux reaction condictions product $1 \mathrm{a}$ is synthesized in $80 \%$ yield.

By reacting equimolar amounts 7-Chloro-4-[(2-hydroxy-benzylidene)-amino]-2-oxo-2H-chromen-3-carbaldehyde and corresponding reagents (according scheme 2) under reflux reaction condictions product $2 \mathrm{a}$ is synthesized in $70 \%$ yield.

By reacting equimolar amounts of 4-[(2-Hydroxy-benzylidene)-amino]-7-(4-hydroxy-phenylamino)-2-oxo-2H-chromen-3carbaldehyde and corresponding reagents (according scheme 3 ) under reflux reaction condictions product $3 a$ is synthesized in $80 \%$ yield.

The structure of 4-Amino-7-chloro -2-oxo-2 $\mathrm{H}$-chromen -3-carbaldehyde derivatives (1a,2a,3a) were determined fromtheir IR, $1 \mathrm{H}$ NMR , 13C NMR spectar and their melting points as follows.

For (1a); IR bands (KBr,cm-1) 3850-2400cm-1 (OH) , NH ; $2910 \mathrm{~cm}-1$ (C-HO stretch.), $1720 \mathrm{~cm}-1$ (C=O) , 1600 (C=C stretch.) , $750 \mathrm{~cm}-1$ (C-H bend.) $600 \mathrm{~cm}-1$ (C-Cl stretch.)

1H NMR (DMSO-d6) סppm ;9.68 ppm s(H,CHO) , 7.21-7.53 t(H,aromatic) , $5.18 \mathrm{~s}(\mathrm{H}, \mathrm{OH}) 4.0$

13 C NMR (DMSO) Dppm ; 166.9ppm (C-Cl), 162ppm (C,COO) ; 152ppm (C,C-O) ; 133.4 (C,C-Cl); $121.7,125.6,128.0$ (3C-aromatic)

For (2a) IR bands (KBr,cm -1) $3400 \mathrm{~cm}-1(\mathrm{OH}) 3200 \mathrm{~cm}-1$ (N-H stretch.) , $3000 \mathrm{~cm}-1$ (C-H stretch.), $3200 \mathrm{~cm} 1$ ( N-H stretch.), $2730 \mathrm{~cm} 1$ (C-H stretch.) , 1725cm1 (C=O stretch.),1600 cm1(C=C stretch.), $1050 \mathrm{~cm} 1$ (C-O stretch), 750cm1(C-H bend.)

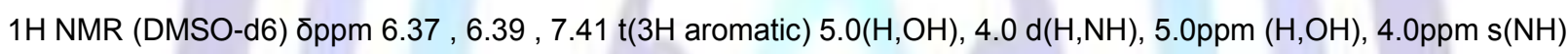

13C NMR (DMSO)סppm181ppm(C,C-NH),178ppm(C,CHO),162ppm (C,COO),151ppm (C,C-O), 105,109 ,116,127ppm (4C aromatic)

For (3a) IR bands (KBr,cm -1) $3280 \mathrm{~cm}-1$ (O-H stretch.),3180cm-1(NH stretch.), $3000 \mathrm{~cm}-1(\mathrm{C}-\mathrm{H}$ stretch.),2400cm-1(O-H carbocylic), $1760 \mathrm{~cm}-1$ (C=O stretch.), $1650 \mathrm{~cm}-1(\mathrm{C}=\mathrm{N}$ stretch), $1710 \mathrm{~cm}-1(\mathrm{C}=\mathrm{O}), 1020 \mathrm{~cm}-1(\mathrm{C}-\mathrm{O}), 750 \mathrm{~cm}-1(\mathrm{C}-\mathrm{H}$ bend.)

1H NMR (DMSO-d6) סppm 7.4 ,6.5,6.4 (3H aromatic), $5.0(\mathrm{H} . \mathrm{OH}), 4.0 \mathrm{~s}(\mathrm{H}, \mathrm{NH}), 3.53 \mathrm{ppm} \mathrm{t}(\mathrm{CH} 2), 2.65 \mathrm{ppm} \mathrm{t}(3 \mathrm{H}, \mathrm{CH} 3 \mathrm{~N})$, $11.40-155 \mathrm{ppm} \mathrm{t}(4 \mathrm{H}, 2 \mathrm{CH} 2)$

13CNMR (DMSO) Dppm 176.0ppm (C,COOH), 167.ppm (C,C-NH), 162.0 (C,C=O), 151.7ppm(C,C-O), 127,109,105ppm (3C aromatic), 51.6(C,C-N), 46.6( C,C-N) , 62.7(C,C-OH), 30.6,27.8ppm (C,CH2)

\section{CONCLUSION}

From the results the followin conclusion were drawn:The study provides the first evidence that compounds (1a,2a,3a) obviously inhibit the growth of S.auerus, E.coli and B.cereus.

The compounds (1a,2a,3a) compared with the antibacterial activity of Streptomycine in S.aureus ,E.coli and B.cereus.

This study provided the first evidence that these compounds 1a,2a,3a showed a significant antibacterial effect against S.aureus,E.coli and B.Cereus.

The chemical structures of synthesizen compounds were determined according to extensive NMR experiments and published data.

\section{Acknowledgements}

The authors thank Prof.Branko Stanovnik,University of Ljubljana and its laboratory staff for 1H NMR spectrum and elemental analyses.

\section{REFERENCES}

1. 1.S.Govori;V.Kalaj;V.Rapic;L.KalajandS.Dakovic,Heterocycel.Commun. 2002.,(8),129

2. 2.B.Stanovnik; H.Susachitzky and E.F.Scriven, Progress in Heterocyclic Chemistry,Pergamon Press ,Oxford, 1993,(5).75-146.

3. S.H.Lee;D.-S.Shin; J.-S,Kim; K.-B. Oh and S.S.Kan, Arch. Pharm. Res 2003., 26.

4. 4.Vyas KB;Nimavat;KS,Jani GR;Hathi MV, 2009)Synthesisi of antimicrobial activity of coumarine derivatives

5. metal complexes:An in vitro evalution.Orbital,(1),183-192.

6. Abyshev AZ; Gimdein Va; Semenov EV ; Agaev EM, Abdulla-zade AA, Gueinov AB,2006 
7. A.Behrami;K.Vaso;I.Krasniqi, J. Int .Environ .Appl.Sci.2010, (5).247 .

8. 7.M.D.Aytemir ; R.C.Hider ; D.D.Erol ; M.Ozalp; and M.Ekizoglu .Turk.J.chem., 2003,445.

9. 8.M.M.El.Saghier;M.B.Naili;B.Kh.Rammash; N.A.Saleh and K.M.Kreddan, Arkivoc, 2007,83.

10. Z.M.Nofal ;M.El-Zahar; and S.Abd El Karim, Molecules, 2000,(5).99 .

11. 10.Chaluvaraju KC and Ishwarbhat K.Asian , J Chem 2008; (20), 4335.

12. 11.Rajan Ra Kali ;Jubie S,Grworamma B; and Suresh B,Asian J Chem 2008:(20), 5289.

13. 12.Ali Mohammed Ashraf ; and Sharayar Mohammed. Boorg Med Chem. Lett 2009;(17),3314.

14. 13.Pandeya SN;Lakshmi VS Aandey A. Indian J Pharma Sci 2003;(65):213

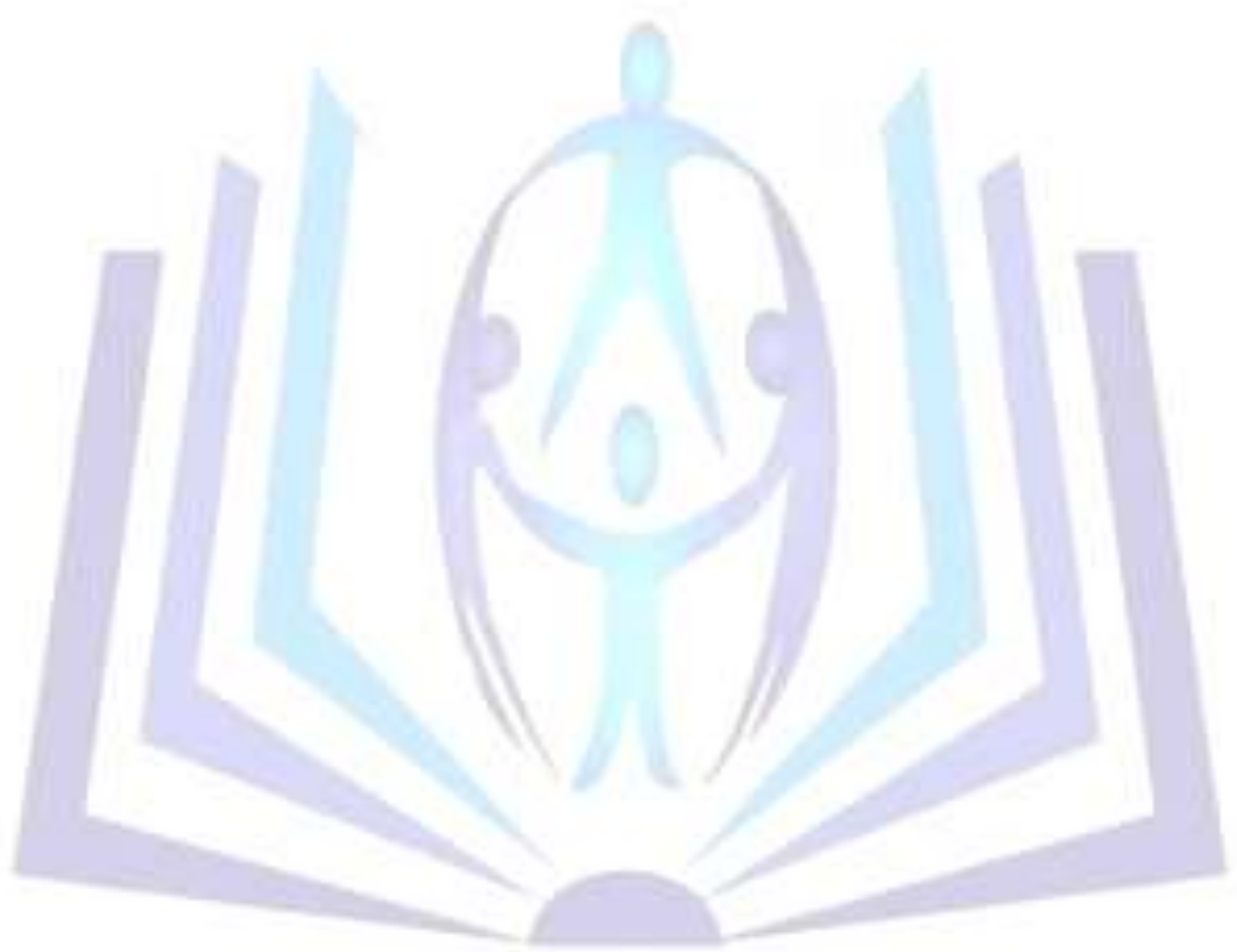

